\title{
Set-Membership Affine-Projection with Estimated Parameters for non-White Inputs
}

\author{
Tadeu N. Ferreira, Markus V. S. Lima, Paulo S. R. Diniz
}

\begin{abstract}
This article proposes versions of the set-membership affine-projection algorithm that estimate an adequate threshold and a proper constraint-vector for a system identification scenario. A novel constraint-vector is proposed for a non-white input, since the Simple-Choice Constraint Vector is asymptotically optimum for white inputs. Non-white inputs are commonplace in communication systems when there is channel encoding or precoding in the transmitter. A fixed and a time-varying threshold set-membership version are used. Simulations show that the proposed algorithm presents lower mean-square error and updates in fewer iterations than the traditional SimpleChoice and Exponential-Decay Constraint Vectors. For the timevarying threshold, the computational complexity of the algorithm is similar to the Simple-Choice Constraint Vector.
\end{abstract}

Keywords-Adaptive filter, set-membership, constraint vector, parameter estimation.

\section{INTRODUCTION}

The emergence of the Affine Projection algorithm (APA) [1] provided a data-reuse alternative to Least-Mean Squares (LMS) providing fast convergence. The price for a faster convergence is a higher computational complexity of APA in comparison to LMS. Then, some data selective algorithms have been developed, such as the Set-Membership Affine Projection (SM-AP) [2],[3], in order to avoid the updates in some iterations. The idea of Set-Membership Filtering (SMF) emerged in [4] with the membership set concept used in system modeling [5]. Both the original Optimal Bounding Ellipsoids (OBE) [5] and the SMF algorithms are based on noise modeling, particularly on previous knowledge of noise power.

The use of the constraint vector (CV) and of a threshold are internal characteristics of the SM-AP algorithm. The SimpleChoice CV (SC-CV) has become the hegemonic CV for the implementation of SM-AP [3], [8]. Recently, other CVs have been proposed to exploit specific characteristics of the problems as in [2], [7]. The development of the Optimal $\mathrm{CV}$ [6] has lead to a possible break of an SC-CV hegemony. Nevertheless, [6] shows that the SC-CV is asymptotically optimal CV for white input signals. The Optimal CV still relies on a predefined threshold, which requires knowledge of the measurement noise.

The use of time-varying thresholds for the SM-AP has its roots in the development of an SM-AP version with constant rate of updates [9]. A time-varying threshold is particularly

Authors thank CNPq, FAPERJ and CAPES for the funding.

T. N. Ferreira, Electrical and Telecom Eng. Post-Grad Program, Universidade Federal Fluminense, Niterói, e-mail: tadeu_ferreira@id.uff.br; M V. S. Lima, P. S. R. Diniz, Electrical Engineering Program, Universidade Federal do Rio de Janeiro, Rio de Janeiro, e-mail: markus.lima@dee.ufrj.br, diniz@smt.ufrj.br. This work has been partially funded by FAPERJ APQ1 E-26/010.001579/2019. interesting if the parameters are estimated, since a proper value of the threshold may be calculated adaptively.

The main proposal of this article is to design SM-AP versions based solely on the estimated parameters. One of the proposed algorithms estimates the adequate threshold for a given input and scenario using the Optimal $\mathrm{CV}$ in few iterations and designing the proper $\mathrm{CV}$ using structures already calculated by the coefficient updates. The other algorithm proposes a time-varying threshold based on the norm of the error vector. A secondary contribution of this article is to develop a CV for non-white input scenarios, such as for channel estimation when a convolutional enconding or a precoding is used in the transmitter.

In this article, Section II describes the SM-AP algorithm and the role of the threshold and the CV. Section III shows the proposed algorithm. In Section IV the main simulation results are presented to validate the proposed algorithm. Section V concludes the article. In this article, minuscule boldface is used for vectors, while capital letter bold symbols are used for matrices.

\section{Set-Membership Affine Projection Algorithm}

Consider a tapped-delay line adaptive filter in the system identification configuration [2]. The input of the adaptive filter is $\mathbf{x}(k) \in \mathbb{R}^{N+1}$ at the $k^{\text {th }}$ discrete sample time, where $\mathbf{x}(k)=\left[\begin{array}{llll}x(k-N) & x(k-N+1) & \ldots & x(k)\end{array}\right]^{T}$. This input $\mathbf{x}(k)$ is also applied to the system whose impulse response $\mathbf{h}(k) \in \mathbb{R}^{N+1}$ is to be identified. The output of the system is $d(k)=\mathbf{x}^{T}(k) \mathbf{h}(k)$ [2]. The adaptive filter is a tappeddelay line characterized by its parameters, i.e., its coefficients, $\mathbf{w}(k) \in \mathbb{R}^{N+1}$. The output of the filter is $y(k)=\mathbf{x}^{T}(k) \mathbf{w}(k)$. The error signal is defined as $e(k)=d(k)-y(k)=d(k)-$ $\mathbf{x}^{T}(k) \mathbf{w}(k)$.

In the APA [2], $L$ previous scalar inputs are used together with the current scalar input $x(k)$, where $L$ is known as data reuse factor. Then the matrix data is $\mathbf{X}(k) \in \mathbb{R}^{(N+1) \times(L+1)}$, where $\mathbf{X}(k)=\left[\begin{array}{llll}\mathbf{x}(k) & \mathbf{x}(k-1) & \ldots & \mathbf{x}(k-L)\end{array}\right]$.

In the SMF approach, the parameter vector $\mathbf{w}(k)$ is estimated with an error which is bounded by a pre-defined threshold $\bar{\gamma}$. The Set-Membership Adaptive Recursive Techniques (SMART) [4] allowed the use of the SMF approach in online applications [6]. At the $k^{\text {th }}$ iteration, a new input vector $\mathbf{x}(k)$ and a sample of the desired signal $d(k)$ are received by the adaptive filter. The pair $(\mathbf{x}(k), d(k))$ generates the constraint set $\mathbb{H}(k)=\left\{\mathbf{w} \in \mathbb{R}^{N+1}:\left|d-\mathbf{w}^{T} \mathbf{x}(k)\right| \leq \bar{\gamma}\right\}$. SM-AP uses the intersection of the previous $L$ constraint sets and the current constraint set, that is, $\Psi_{k-L}^{k} \triangleq \bigcap_{i=k-L}^{k} \mathbb{H}(i)$. At each iteration, SM-AP chooses a new parameter vector $\mathbf{w}(k+1)$ 
belonging to $\Psi_{k-L}^{k}$, which is the closest one to $\mathbf{w}(k)$ for the SC-CV. In summary, SM-AP minimizes $\|\mathbf{w}(k+1)-\mathbf{w}(k)\|^{2}$ subjected to $\mathbf{w}(k+1) \in \Psi_{k-L}^{k}[3],[6]$.

\section{A. Threshold and the Constraint-Vector}

An important definition to the SM-AP algorithm is the constraint set, which is defined as $\mathbb{H}(k)=\left\{\mathbf{w} \in \mathbb{R}^{N+1}\right.$ : $\left.\left|d(k)-\mathbf{w}^{T}(k) \mathbf{x}(k)\right| \leq \bar{\gamma}\right\}$ [6], where $\bar{\gamma}$ is the threshold. Therefore, if $\left|d(k)-\mathbf{w}^{T}(k) \mathbf{x}(k)\right| \leq \bar{\gamma}$ already holds for the current coefficient vector, then it is not going be updated, i.e. $\mathbf{w}(k+1)=\mathbf{w}(k)$.

A cost function often used is $\|\mathbf{w}(k+1)-\mathbf{w}(k)\|^{2}$, which is minimized subjected to $\mathbf{d}(k)-\mathbf{X}^{T}(k) \mathbf{w}(k)=\gamma$, where $\gamma$ is the CV. The CV defines the subset of $\Psi_{k-L}^{k}$ where the projection is performed [7]. The coefficient updates in SM-AP is given by:

$$
\begin{aligned}
& \mathbf{w}(k+1)= \\
& \begin{cases}\mathbf{w}(k)+\mathbf{X}\left(\delta \mathbf{I}+\mathbf{X}^{T} \mathbf{X}\right)^{-1}(\mathbf{e}(k)-\gamma(k)) & |e(k)|>\bar{\gamma} \\
\mathbf{w}(k) & |e(k)| \leq \bar{\gamma}\end{cases}
\end{aligned}
$$

where $\mathbf{e}(k)=\mathbf{d}(k)-\mathbf{X}^{T}(k) \mathbf{w}(k)$ and $\delta$ is a regularization parameter. Parameter $\bar{\gamma}$ is typically set to $\sqrt{\tau \sigma_{n}^{2}}$ [2], where $\tau$ is usually chosen as 4 or 5 and $\sigma_{n}^{2}$ is the measurement noise variance, which is assumed to be known. In this paper, an estimation of the measurement noise is used.

Several CV $\gamma(k)$ exists [2]. The most popular $\mathrm{CV}$ is the Simple Choice CV (SC-CV), defined as $\gamma_{S C}(k)=$ $\left[\begin{array}{llll}\bar{\gamma} \operatorname{sign}(e(k)) & e(k-1) & \ldots & e(k-N)\end{array}\right]^{T}$ [2]. In [6], the $\mathrm{SC}-\mathrm{CV}$ is proved to be asymptotically optimal for the white input case.

\section{B. Optimal Constraint-Vector}

Optimal CV [6] finds the CV that minimizes the SM-AP problem formulated as:

$$
\begin{array}{rc}
\min _{\boldsymbol{\gamma}, \mathbf{w}(k+1)} & \|\mathbf{w}(k+1)-\mathbf{w}(k)\|^{2}+\frac{1}{\delta}\left\|\mathbf{d}(k)-\mathbf{X}^{T}(k) \mathbf{w}(k)\right\|, \\
\text { s. t. } & \|\gamma(k)\|_{\infty} \leq \bar{\gamma}
\end{array}
$$

Eq. (2) allows as possible results the CVs whose elements are less than the threshold $\bar{\gamma}$, which leads to the use of the original constraint $\mathbf{w}(k+1) \in \Psi_{k-L}^{k}$ of the SM-AP.

In general, the choice of $\bar{\gamma}$ and $\gamma(k)$ in SM-AP is empirical. The Optimal CV [6] leads to a more mathematical approach, but it presents a high computational burden.

The use of the Optimal CV generates CVs whose largest element may be smaller than the pre-defined $\bar{\gamma}$. It indicates that the modeling for $\bar{\gamma}$ may be changed based on the Optimal CV. The choice of an Optimal CV may not lead to a projection which has a distance of $\bar{\gamma}$ from the a posteriori null error which is the solution to the SM-APA cost function. On the other hand, if a $\hat{\bar{\gamma}}$ may be selected as the largest element of the Optimal CV, restoring the property of the SM-APA solution. Then, the Optimal CV indirectly provides an estimation of the threshold $\bar{\gamma}$.

\section{Proposed Algorithms}

Our objective is to estimate $\bar{\gamma}$ and then build a CV. The $\mathrm{CV}$ generation also uses the estimation of how far from the white distribution the input is. This is performed by using a time-average estimate of the correlation of the input signal. A rough instantaneous estimation of the correlation is already performed in the APA by calculating $\mathbf{X}^{T}(k) \mathbf{X}(k)$.

Using the Optimal CV is a problem due to an inherent high computational complexity and to the nature of SM-AP, which calculates Optimal CV if the $\bar{\gamma}$ is high enough. Then, an underestimated $\bar{\gamma}$ would not be detected. Our strategy aims to circumvent both issues. The algorithm is divided in two stages: a short training stage and a large execution stage. During training, the update is performed by APA, but the Optimal $\mathrm{CV}$ is also calculated, and the input correlation is estimated. After training, $\bar{\gamma}$ is estimated by $\left\|\gamma_{m}\right\|_{\infty}$, where $\gamma_{m}$ is the median of each element along the training, obtained by the Optimal CV. The CV building is a more complicated issue.

\section{A. Building a Constraint-Vector}

One of the first SM algorithms was the SM-NLMS algorithm [4]. Defining SM-NLMS does not lead to creating a CV. SM-NLMS uses a scalar error, which is compared to $\bar{\gamma}$ at each iteration. Moreover, the coefficient vector is updated by using $\bar{\gamma} \operatorname{sign}(e(k))$.

The emergence of SM-AP [3] and SM Binormalized NLMS (SM-BNLMS) [8] leads to the creation of the CV for the SM algorithms. The solution, which is still placed at $\bar{\gamma} \operatorname{sign}(e(k))$ instead of $e(k)$, is advantageous since the $\mathbf{w}(k+1)$ is updated inside the current constraint set while generating the minimum distortion to the original error, because it is placed on the edge of $\Psi_{k-L}^{k}$. The other entries of the CV are chosen not to change the current a posteriori error. Then,

$$
\gamma_{S C}(k)=\left[\begin{array}{c}
\bar{\gamma} \operatorname{sign}(e(k)) \\
e(k-1) \\
\vdots \\
e(k-N)
\end{array}\right]
$$

Eq. (3) leads to the update based on the vector $\mathbf{e}(k)-$ $\gamma(k)=\left[\begin{array}{llll}\bar{\gamma} \operatorname{sign}(e(k)) & 0 & \ldots & 0\end{array}\right]^{T}$. Due to the SM-AP's good performance with $\mathrm{SC}-\mathrm{CV}$, it is the most popular version of the SM-AP nowadays. Recently, the optimality of SC-CV for orthogonal inputs and for white signals (in the mean) has been shown [6]. Then, some design guidelines may be developed:

- The CV should converge to the SC-CV for white inputs;

- The first component of the CV should be $e(k)-$ $\bar{\gamma} \operatorname{sign}(e(k))$, which is the choice of the large majority of the existing $\mathrm{CV}$ [2].

There is a wide variety of non-white signals, which makes it harder to design proper CVs. The nullity of almost all of the elements in $\mathbf{e}(k)-\gamma(k)$ for the SC-CV removes the effects of $e(k-1), \ldots, e(k-L)$ in the coefficient updates. The inclusion of the effects of all elements, as in the Fixed-Modulus Error Bound (FMEB) CV [2], in the Trivial Choice (TC) CV [2] or in the Exponentially-Decay (ED) CV [7] resulted in worse performance when compared to the SC-CV in some scenarios. 
The inclusion of the effect of some of the elements of $\mathbf{e}(k)-$ $\gamma(k)$ may be a possible solution. Our proposal is to include only the effects of $e(k)$ and $e(k-1)$. The proposed $\mathrm{CV}$ is then configured as:

$$
\gamma(k)=\left[\begin{array}{c}
e(k)-\bar{\gamma} \operatorname{sign}(e(k)) \\
e(k-1)-\alpha(k) \operatorname{sign}(e(k-1)) \\
e(k-2) \\
\vdots \\
e(k-L)
\end{array}\right] .
$$

The value of $\alpha(k)$ is used based on the $(2,1)$ entry of the estimated correlation matrix, since $e(k-1)=d(k-1)-$ $\mathbf{w}^{T}(k-1) \mathbf{x}(k-1)$, and the $(2,1)$ estimates the correlation between $\mathbf{x}(k-1)$ and $\mathbf{x}(k)$.

After some experimental tests, $\alpha$ was chosen as $\left|r_{2,1}\right| / 5$, where $r_{2,1}$ is the $(2,1)$ entry of the estimated correlation matrix.

Keep in mind that the threshold $\bar{\gamma}$ used in our proposed algorithm is also estimated from the training part, using $\left\|\gamma_{m}\right\|_{\infty}$, with $\gamma_{m}$ as the median of each element along the training. Median eliminates possible outliers in the optimal CV estimation. This idea is inspired by the time-varying threshold used in [10]. The whole proposed algorithm is summarized in Table I.

TABLE I

PROPOSED ALGORITHM WITH FIXED THRESHOLD.

\begin{tabular}{l}
\hline Stage 1: Training \\
\hline Coeffient updates using APA; \\
Correlation matrix estimate updates using $\mathbf{X}^{T}(k) \mathbf{X}(k) ;$ \\
$\bar{\gamma}$ estimate updates using $\left\|\gamma_{o p t}(k)\right\|_{\infty}$ \\
\hline Stage 2: Running \\
\hline Coefficient updates using the proposed SM-AP; \\
$\bar{\gamma}$ defined by the estimate of the training stage; \\
CV defined in (4), based on the training stage. \\
\hline
\end{tabular}

\section{B. Time-Varying Threshold}

Another approach for performing the estimation of the SM threshold is to use a time-varying threshold. The proposed estimation is inspired by the time-varying threshold [11], by avoiding the term containing $\sigma_{n}^{2}$, which requires previous knowledge of the measurement noise variance. The estimated threshold $\hat{\bar{\gamma}}(k)$ at iteration $k$ is given by:

$$
\hat{\bar{\gamma}}(k)=\lambda \hat{\bar{\gamma}}(k-1)+(1-\lambda) \epsilon\|\mathbf{e}(k)\|,
$$

where $\lambda$ is a forgetting factor and $\epsilon$ is a scaling factor for adjusting the proportionality between $\hat{\bar{\gamma}}(k)$ and $\|\mathbf{e}(k)\|$. In our simulations, $\hat{\bar{\gamma}}(0)=0$.

A summary of the proposed algorithm with a time-varying thresold is presented in Table II.

\section{Remarks on Convergence}

The proposed CV defined in Eq. (4) shows an intermediary behavior between the SC-CV and the Fixed Modulus ErrorBound (FMEB) CV [2] [7], since the first element of the proposed $\mathrm{CV}$ is identical to both $\mathrm{SC}-\mathrm{CV}$ and FMEB $\mathrm{CV}$, the
TABLE II

PROPOSED ALGORITHM WITH TIME-VARYING THRESHOLD.

\begin{tabular}{l}
\hline Stage 1: Training \\
\hline Coeffient updates using SM-AP; \\
Correlation matrix estimate updates using $\mathbf{X}^{T}(k) \mathbf{X}(k) ;$ \\
$\bar{\gamma}$ estimate updates using Eq. (5), starting with $\hat{\bar{\gamma}}(0)=0$ \\
\hline Stage 2: Running \\
\hline Coefficient updates using the SM-AP with the proposed CV; \\
$\bar{\gamma}$ estimate updates using Eq. (5). \\
CV defined in (4), based on the training stage. \\
\hline
\end{tabular}

second element combines the weighted FMEB CV and the SC$\mathrm{CV}$, while the others are identical to the SC-CV corresponding element. Then, the same reasoning applied to the convergence of ED (Exponentially Decay) CV in [7] may also be applied here to the proposed $\mathrm{CV}$.

The proposed $\mathrm{CV}$ is also a possible solution to Eq. (2) of the Optimal CV. Then, the reasoning used in [6] to address the convergence of the Optimal CV may also be applied here to the special case of a fixed $\mathrm{CV}$ inside the feasible set of solutions to Eq. (2).

The use of a time-varying threshold may present some concerns over convergence, since a small rate of updates in the beginning may slow the convergence down. Nevertheless, our algorithm starts with a zero threshold, that is, every iteration presents an update, with a slow increase in its value until a proper estimate for the threshold is reached.

\section{Computational Complexity Remarks}

As presented in [12], the asymptotic computational complexity of the Optimal CV lies between $\mathcal{O}\left(L^{3.5}\right)$ and $\mathcal{O}\left(L^{4}\right)$, during the training stage. On the other hand, the computational complexity of a pre-defined $\mathrm{CV}$ is $\mathbf{O}(L)$ after the training stage. In our proposed algorithm with fixed $\mathrm{CV}$, the number of training samples is much smaller than the number of samples. In Section IV, the training part comprises 20 samples out of 3,500. Besides that, the proposed fixed $\mathrm{CV}$ requires fewer updates than the SC-CV.

For the proposed algorithm with a time-varying threshold, the Optimal CV is not used in the training part. The estimated threshold is updated in every iteration using Eq. (5). A summary of the asymptotic computational complexity of the proposed $\mathrm{CV}$ s for the SM-AP with $\mathrm{SC}-\mathrm{CV}$ is presented in Table III.

TABLE III

COMPARISON FOR THE ASYMPTOTIC COMPUTATIONAL COMPLEXITY OF THE PROPOSED CVS AND THE SM-AP WITH SC-CV.

\begin{tabular}{cc}
\hline Version of the SM-AP & Complexity \\
\hline SC-CV & $\mathcal{O}(L)$ \\
Proposed Fixed CV & $\mathcal{O}\left(L_{T}^{3.5}\right)$ then $+\mathcal{O}(L)$, \\
Proposed Time-Varying CV & $\mathcal{O}(L)$ \\
\hline
\end{tabular}

\section{RESULTS}

This section presents the results of computer simulations for the performance comparison of the proposed CVs, presented in 
Section III, compared to SM-AP with SC-CV [2] and ED-CV. Besides that, the fixed threshold algorithm is also simulated in a situation where the parameters were known. Simulations have been performed on Matlab software in a Ubuntu Linux distribution.

The algorithms are compared in terms of mean-squared error (MSE), that is, $\sum_{m=0}^{M-1} \sum_{k=0}^{K-1}\left|e_{m}(k)\right|^{2}$, where $M=600$ is the number of Monte-Carlo runs and $K=3,500$ is the amount of transmitted symbols, and $e_{m}(k)$ is the error at the $m$-th MonteCarlo run. Only 20 symbols are used in the training phase. Another comparative figure-of-merit used in the simulations is the Misalignment $\phi(k)$, defined in Eq. (6).

$$
\phi(k)=\frac{1}{M} \sum_{m=0}^{M-1} \sum_{k=0}^{K-1} \frac{\left\|\mathbf{w}_{m}(k)-\mathbf{h}(k)\right\|_{2}^{2}}{\left\|\mathbf{w}_{m}(k)\right\|_{2}^{2}},
$$

where $\mathbf{w}_{m}(k)$ is the coefficient vector in the $m$-th MonteCarlos run, and $h$ is the impulse response of the channel, which has all the $N=16$ taps equal to one during the first 200 iterations and then all of them change to 2 .

As presented in [6], the SC-CV is the Optimal CV in the mean for the white input. Our focus in the simulations are the colored-input scenarios.

In the first experiment, a $4^{\text {th }}$ order auto-regressive (AR) is used to generate the adaptive filter input signal as $\mathbf{x}(n)=$ $-0.95 \mathbf{x}(n-1)-0.19 \mathbf{x}(n-2)-0.09 \mathbf{x}(n-3)+0.5 \mathbf{x}(n-$ $4)+\mathbf{n}(k)$. The MSE results for the first experiment are shown in Fig. 1. The proposed algorithm with fixed threshold presents a lower final MSE. Note also that the training part comprises only 20 samples. The performance is not degraded even when there is a change to the impulse response at the 200-th iteration. For the time-varying threshold, the forgetting factor is set as $\lambda=0.999$ and $\epsilon=0.75$.

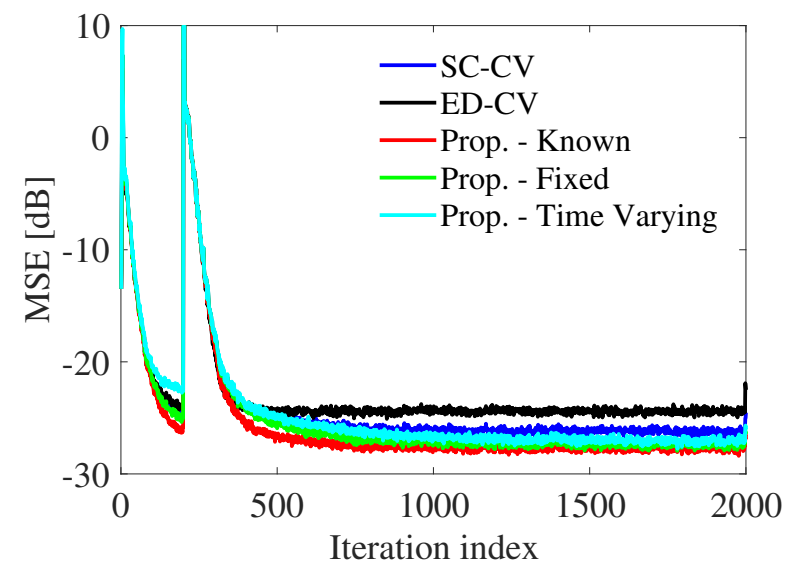

Fig. 1. MSE for an AR-4 process input.

In the same simulation, the misalignment was also measured. The results are shown in Fig. 2. The proposed algorithm with a fixed threshold presents a lower final misalignment. The parameter $\epsilon$ of the proposed time-varying threshold was reset as $\epsilon=1$.

Another simulation was performed using an AR-1 input, generated by $\mathbf{x}(n)=-0.95 \mathbf{x}(n-1) \mathbf{n}(k)$. Such a scenario

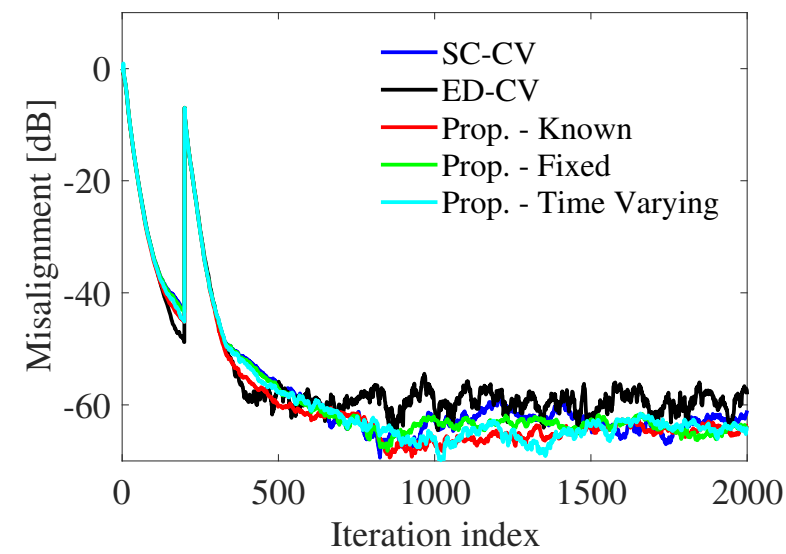

Fig. 2. Misalignment for an AR-4 process input.

tends to be more favorable to the $\mathrm{SC}-\mathrm{CV}$, as shown in [6]. The results are presented in Fig. 3.

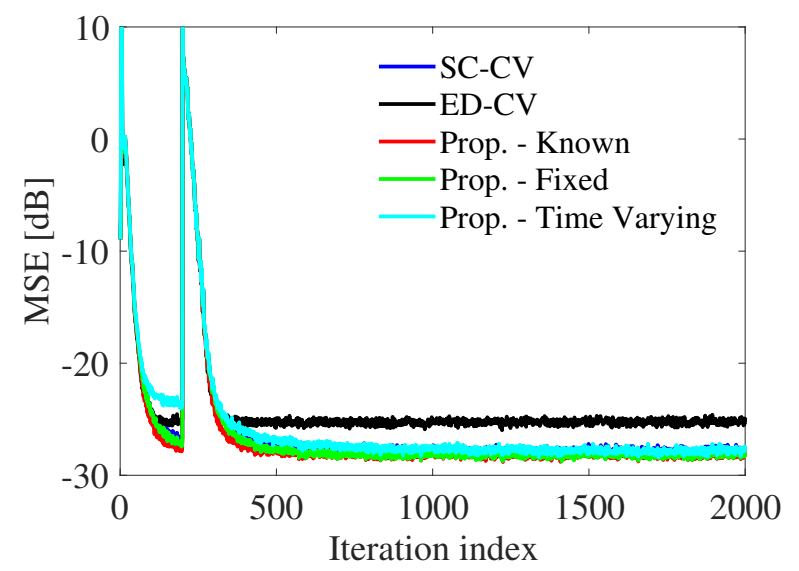

Fig. 3. MSE for an AR-1 process input.

One may realize in Fig. 3 that the proposed algorithm with time-varying threshold presents a slower convergence than the proposed fixed threshold, as well as the SC-CV and ED-CV.

Fig. 4 shows the results for the misalignment in the AR1 simulated environment. One observes that the results are equivalent for all the CVs, except for the ED-CV, which has the worst performance.

Since SM algorithms do not update in every iteration, Table IV shows the update rate for each algorithm, defined as the ratio of the number of iterations with updates to the number of updates where the algorithm runs. For an AR-4 input, the proposed algorithm with a fixed threshold, then the algorithm's training part is not considered in the ratio since an affine projection update is used.

The proposed algorithm with fixed threshold updates in a smaller number of iterations compared to the SC-CV. The proposed algorithm with time-varying threshold presents an intermediary rate of iterations when compared to SC-CV and ED-CV for an AR-4 input. On the other hand, the version with a time-varying threshold presents a lower complexity for the update than the fixed threshold version, as shown 


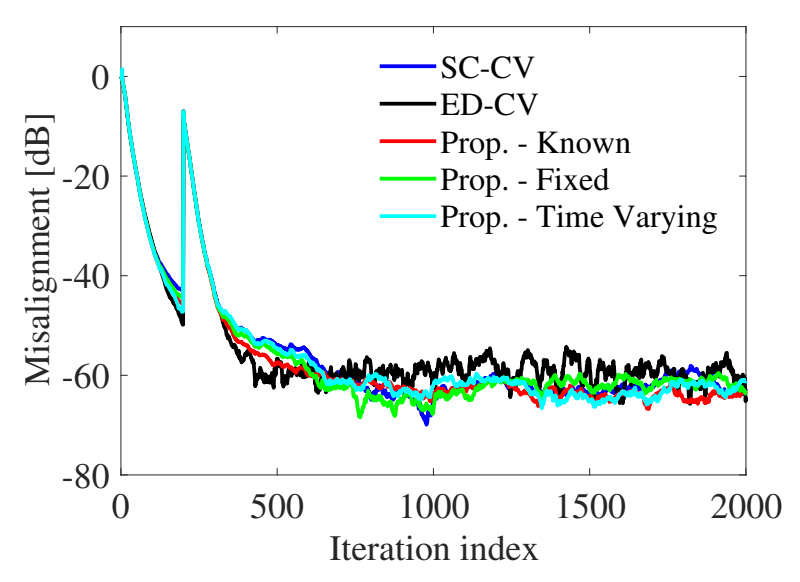

Fig. 4. Misalignment for an AR-1 process input.

TABLE IV

SM UPDATE RATES FOR EACH ALGORITHM IN EACH OF THE SCENARIOS.

\begin{tabular}{|c|c|c|c|c|}
\hline CV & \multicolumn{4}{|c|}{ Update Rate } \\
\hline & AR4 & AR1 & White & Rep. Code \\
\hline SC-CV & 0.271 & 0.187 & 0.156 & 0.193 \\
\hline ED-CV & 0.335 & 0.281 & 0.203 & 0.181 \\
\hline Proposed: Fixed & 0.215 & 0.165 & 0.151 & 0.258 \\
\hline Proposed: Time-Varying & 0.304 & 0.168 & 0.150 & 0.165 \\
\hline
\end{tabular}

in Section III-D. Then, the proposed fixed threshold version presents lower MSE, a slightly lower misalignment, and fewer updates than the SC-CV by using only 20 samples of training. The proposed time-varying threshold version presents lower MSE and misalignment, with an update rate greater than SC$\mathrm{CV}$, but smaller than ED-CV.

In the case of AR-1 input, both proposed versions of SM-AP present a smaller update rate than SC-CV and ED-CV.

In our simulations, we also plotted the proposed algorithm with a fixed threshold considering the situation where the parameters were all known. As can be seen in Figs. 1 and 2, the version with known parameters presents a lower MSE and misalignment. Then, we conclude that the performance of the proposed algorithm presents a sub-optimal estimation.

It is remarkable that the training of the proposed fixed $\mathrm{CV}$ is performed only once, even if the impulse response is changed, as depicted in Figs. 1-4. On the other hand, if the input signal changes its input statistics, the adaptive filter should be retrained.

The proposed algorithm was also tested in a scenario where a simple channel encoding known as repetition code [13] with length 4 was used. The Misalignment for the proposed algorithm as well as the SC-CV and ED-CV is shown in Fig. 5. The update rates are also shown in Table IV.

\section{CONCLUSion}

This article describes two versions of the SM-AP algorithm solely based on estimation. One of them estimates the CV parameters based on the optimal CV. The other one is based on a time-varying estimation of the noise power for the SM threshold. Simulation results show that the proposed algorithm outperforms the benchmarks SC-CV and ED-CV in terms of mean-squared error with less update, with the exception of the scenario with repetition code, where the fixed version presented more updates. Another contribution of this article is that the proposed $\mathrm{CV}$ is developed mainly for non-white inputs, which is common when the transmitter has an encoder.

\section{ACKNOWLEDGEMENT}

The authors would like to thank CNPq, CAPES, and FAPERJ agencies for funding. This work is funded by FAPERJ E-26/010.001579/2019, E-26/210.524/2019 (248292).

\section{REFERENCES}

[1] K. Ozeki, T. Umeda, "An adaptive filtering algorithm using an orthogonal projection to an affine subspace and its properties," Electronics and Communications in Japan, vol. 67-A, n. 5, pp. 19-27, 1984.

[2] P. S. R. Diniz, Adaptive Filtering: Algorithms and Practical Implementation, 5th Ed., Springer, Cham, 2019.

[3] S. Werner, P. S. R. Diniz, "Set-membership affine projection algorithm," IEEE Sig. Processing Letters, vol. 8, n. 8, pp. 231-235, Aug. 2001.

[4] S. Gollamudi, S. Nagaraj, S. Kapoor, Y. F. Huang, "Set-membership filtering and a set-membership normalized LMS algorithm with an adaptive step size," IEEE Sig. Processing Letters, vol. 5, n. 5, pp. 111114, May 1998.

[5] A. Rao, Y. F. Huang, "Tracking characteristics of an OBE parameterestimation algorithm," IEEE Trans. Sig. Processing, vol. 41, n. 3, pp. 1140-1448, Mar. 1993.

[6] W. A. Martins, M. V. S. Lima, P. S. R. Diniz, T. Ferreira, "Optimal constraint vectors for set-membership affine projection algorithms," Signal Processing, vol. 134, pp. 285-294, May 2017.

[7] M. V. S. Lima, T. Ferreira, W. A. Martins, P. S. R. Diniz, "Sparsity-aware data-selective adaptive filters," IEEE Trans. Signal Processing, vol. 62, n. 17, pp. 4557-4572, July 2014.

[8] P. S. R. Diniz, S. Werner, "Set-membership binormalized data-reusing LMS algorithms," IEEE Trans. Sig. Processing, vol. 51, n. 1, pp. 124134, Jan. 2003.

[9] J. Galdino, J. A. Apolinário Jr., M. L. R. de Campos, "A Set-Membership NLMS Algorithm withTime-Varying Error Bound," Proc. of IEEE International Symp. on Circuit and Systems, pp. 277-280, Kos, May 2006.

[10] M. Z. A. Bhotto, A. Antoniou, "A robust constrained set-membership affine-projection adaptive-filtering algorithm," Proc. of International Symposium on Communications, Control and Signal Processing, pp. 1-4, Rome, May 2012.

[11] R. C. de Lamare, P. S. R. Diniz, "Set-Membership Adaptive Algorithms based on Time-Varying Error Bounds and their Application to Interference Suppression," in Proc. of ITS 2006, pp. 563-567, Fortaleza, Sept 2006.

[12] T. Ferreira, W. A. Martins, M. V. S. Lima, P. S. R. Diniz, "Convex combination of constraint vectors for set-membership affine projection algorithms," in Proc. of the IEEE ICASSP, pp. 4858-4862, Brighton, UK, May 2019.

[13] T. Cover, J. Thomas, Elements of Information Theory, 2nd Edition, Wiley, 2006.

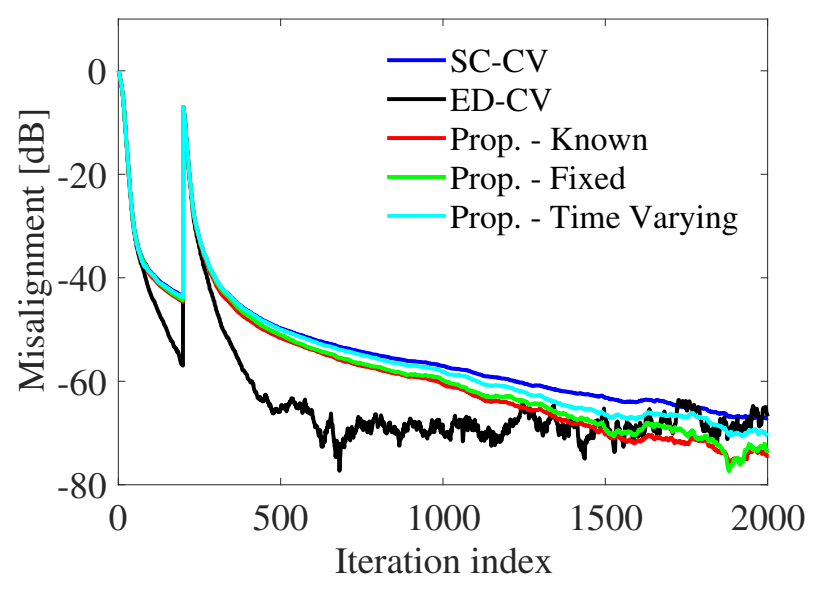

Fig. 5. Misalignment for the scenario with Repetition Code [13]. 